\title{
The chemokine receptor CXCR3 limits injury after acute toxic liver damage
}

\author{
Mirko Moreno Zaldivar ${ }^{1}$, Marie-Luise Berres ${ }^{1}$, Hacer Sahin ${ }^{1}$, Andreas Nellen ${ }^{1}$, Daniel Heinrichs ${ }^{1}$, Petra Schmitz ${ }^{1}$, \\ Nicolaus Gassler ${ }^{2}$, Konrad L Streetz ${ }^{1}$, Christian Trautwein ${ }^{1}$ and Hermann E Wasmuth ${ }^{1}$
}

Although acute liver failure is a rare disease, its presence is associated with high morbidity and mortality in affected patients. While a contribution of the immune system to the outcome of toxic liver failure is anticipated, functionally relevant immune cell receptors for liver cell damage need to be better defined. We here investigate the relevance of the chemokine receptor CXCR3, which is important for hepatic immune cell infiltration, in a model of experimental acute liver failure. Liver injury was induced by a single intraperitoneal injection of carbon tetrachloride $\left(\mathrm{CCl}_{4}\right)$ in $\mathrm{CXCR}^{-/-}, \mathrm{CCR}^{-/-}$, $\mathrm{CCR}^{-1-}$ and wild-type mice. In this model, $\mathrm{CXCR}^{-/-}$mice displayed augmented liver damage compared with all other mouse strains as assessed by liver histology and serum transaminases 24 and $72 \mathrm{~h}$ after injury. Phenotypically, CXCR3 ${ }^{-1-}$ mice had significantly reduced intrahepatic NK and NKT cells after injury at all investigated time points (all $P<0.05$ ), but strongly elevated expression levels of IL1- $\beta$, TNF- $\alpha$ and IFN- $\gamma$. In line with a functional role of innate immune cells, wildtype mice depleted for NK cells with an anti-ASIALO GM1 antibody before liver injury also displayed increased liver injury after $\mathrm{CCl}_{4}$ challenge. $\mathrm{CXCR}^{-1-}$ and NK cell-depleted mice show reduced apoptotic liver cells (TUNEL assay), but more necrotic hepatocytes. Functionally, the augmented liver cell necrosis in $\mathrm{CXCR3}^{-1-}$ and NK cell-depleted mice was associated with increased expression of high mobility group 1 (HMGB1) protein and a consecutive enhanced infiltration of neutrophils into the liver. In conclusion, the results demonstrate a primarily unexpected beneficial role of CXCR3 in acute toxic liver injury. These findings should be taken into account when planning trials with CXCR3 antagonists.

Laboratory Investigation (2012) 92, 724-734; doi:10.1038/labinvest.2012.48; published online 19 March 2012

KEYWORDS: chemokine receptor; CXCR3; HMGB1; immune cells; liver failure; neutrophils

Although liver failure is a quite rare clinical condition, its appearance is associated with high mortality and morbidity in affected individuals. The main identifiable causes of acute liver failure are drugs, toxins and viral infections. ${ }^{1}$ From a pathophysiological point of view, acute toxic liver failure results from a massive necrosis of hepatocytes that provokes a strong inflammatory immune response within the liver. ${ }^{2}$ Upon activation, liver resident and infiltrating immune cells (including T cells, NK cells and NKT cells) secrete diverse proinflammatory chemokines and cytokines, including interferon (IFN)- $\gamma^{3,4}$ which perpetuate liver cell damage. IFN- $\gamma$ in turn strongly activates the transcription of the chemokines CXCL9, CXCL10 and CXCL11. ${ }^{5}$ The canonical receptor for all three chemokines is CXCR $3,{ }^{6}$ which is expressed on various cell sub-populations within the liver, including liver endothelial cells, stellate cells, T cells, NK cells ${ }^{7}$ and NKT cells. ${ }^{8}$ The interaction between these three chemokines and their receptor mediates the attachment of these immune cells to endothelial cells ${ }^{9}$ and thus appears to be crucial for the recruitment of T, NK and NKT cells into the liver. ${ }^{10,11}$ Activated hepatic NKT cells by themselves also secrete large amounts of IFN- $\gamma$, which in turn promotes the expression of CXCL10 and leads to hepatic recruitment of CXCR3 expressing regulatory $\mathrm{T}$ cells $\left(\mathrm{T}_{\mathrm{REG}}\right)^{12}$

However, despite accumulating evidence for the role of CXCR3 in the hepatic immune response, the specific contribution of this chemokine receptor and its ligands CXCL9, 10 and 11 to acute liver injury has not yet been finally elucidated in vivo. A recent study demonstrated that adenoviral infection increases intrahepatic IFN- $\gamma$ levels, which lead to enhanced CXCL9 expression and an elevated numbers of CXCR3 $+\gamma \delta \mathrm{T}$ cells within the livers, causing acute liver damage. ${ }^{13}$ Another study dealt with the impact of CXCR3 on liver ischemia/reperfusion injury (IRI) in rats. ${ }^{14}$ Zhai et $a l^{14}$

\footnotetext{
${ }^{1}$ Medical Department III, University Hospital Aachen, Aachen, Germany and ${ }^{2}$ Institute of Pathology, University Hospital Aachen, Aachen, Germany Correspondence: Professor HE Wasmuth, MD, Medical Department III, University Hospital Aachen (UKA), RWTH Aachen, Pauwelsstrasse 30, 52074 Aachen, Germany. E-mail: hwasmuth@ukaachen.de
} 


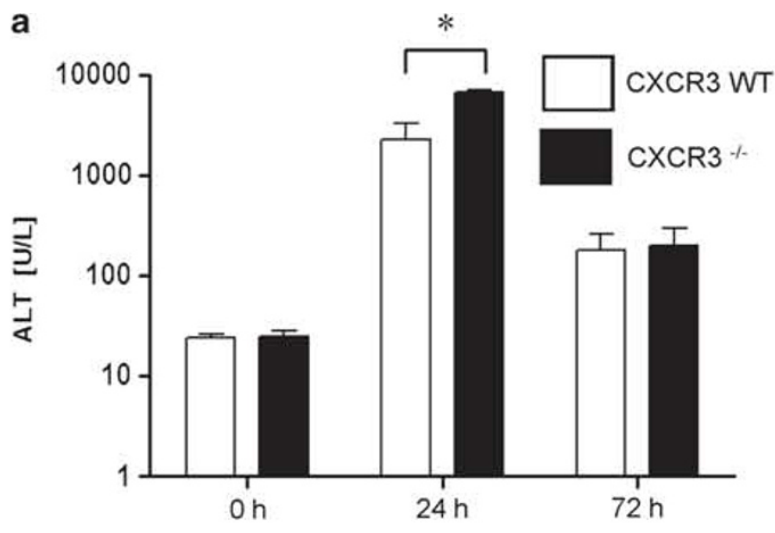

b
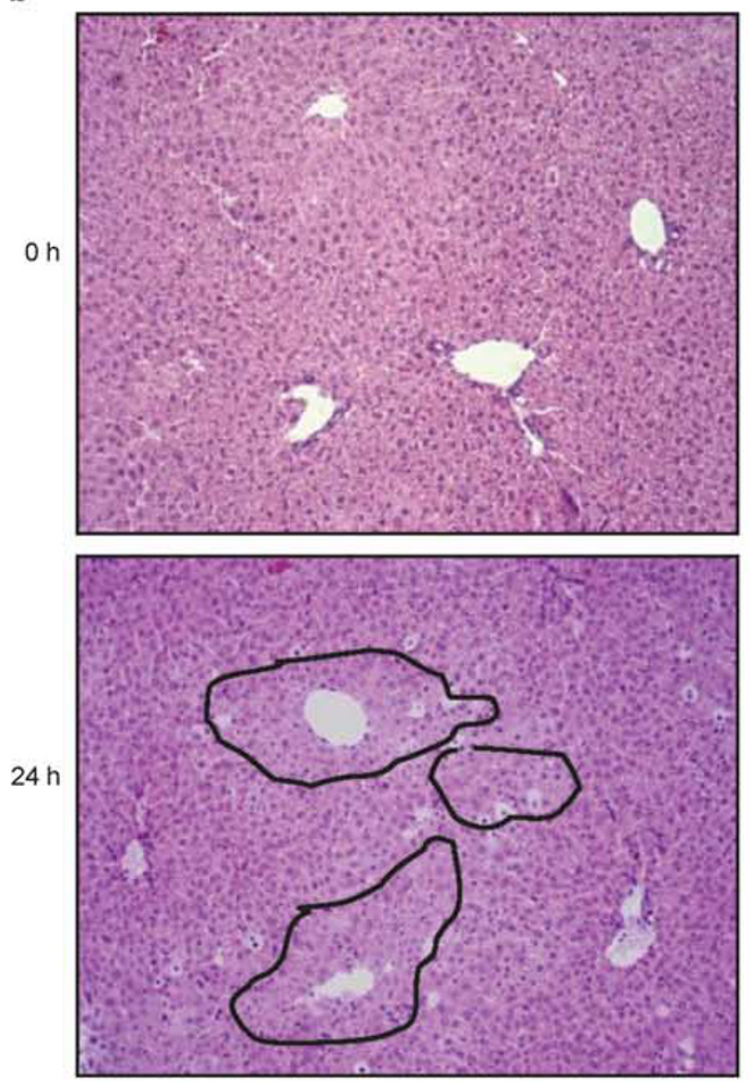

$72 \mathrm{~h}$

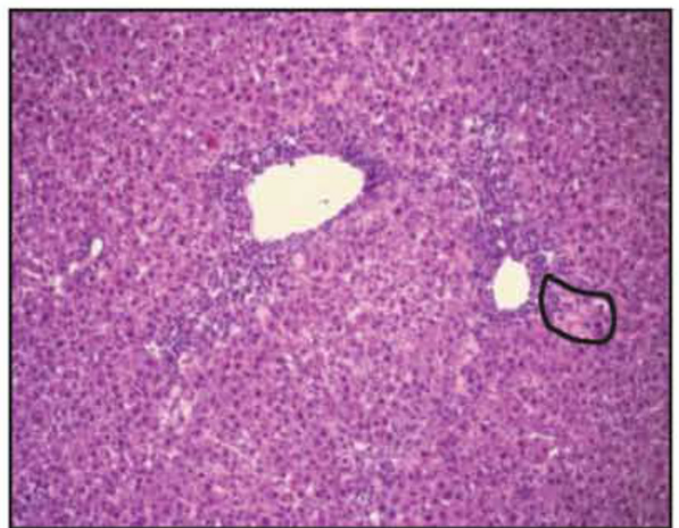

$\mathrm{CXCR} 3^{-1}$
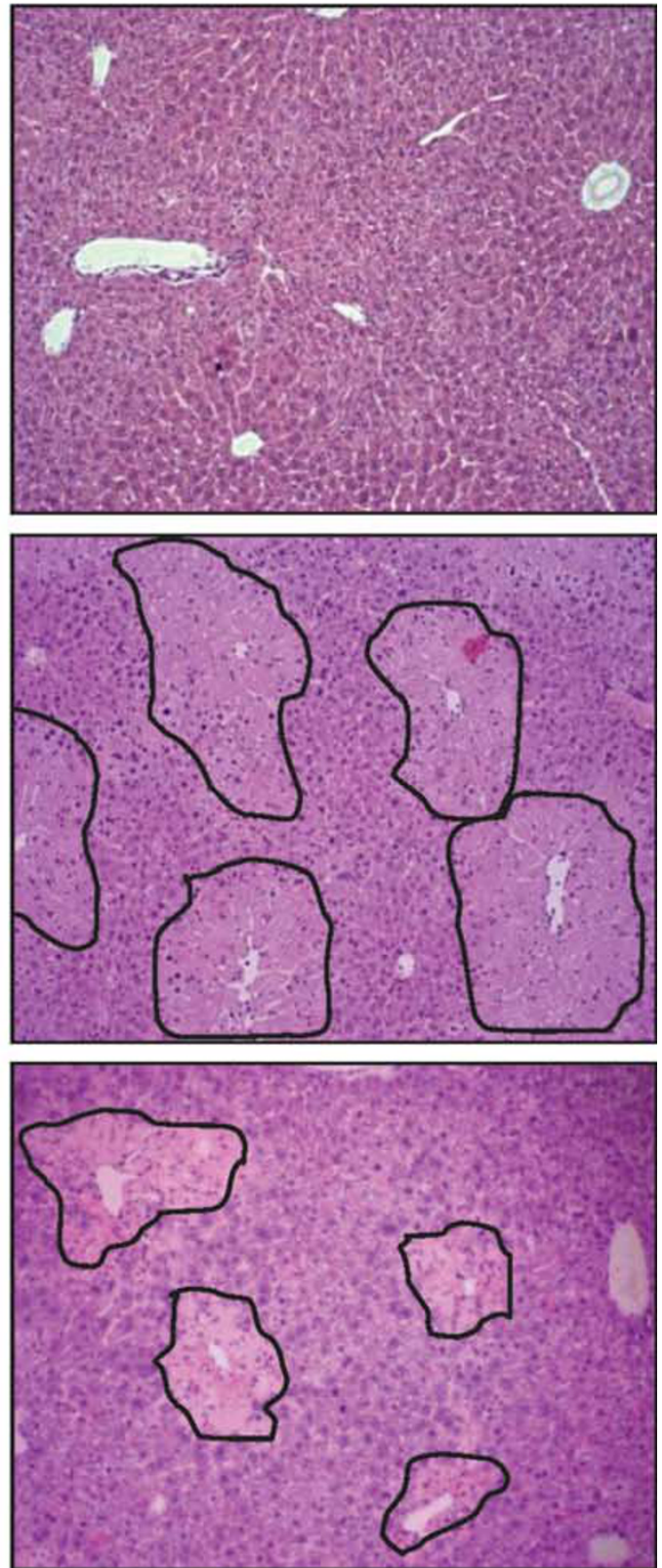

Figure 1 For caption see page 726. 
observed that IRI triggered an increased intrahepatic expression of the chemokines CXCL9, 10 and 11. This enhanced expression resulted in a selective recruitment of $\mathrm{CXCR}^{+}{ }^{+} \mathrm{CD}^{+}{ }^{+} \mathrm{T}$ cells. Conversely, CXCR3 receptor blockade ameliorated hepatocellular damage accompanied by a reduction of intrahepatic CXCR3 ${ }^{+} \mathrm{T}$ cells. In contrast to these potentially detrimental effects of CXCR3, in Concanavalin A (ConA)-induced liver injury CXCR3 appears to be essential for the recruitment of $\mathrm{T}_{\mathrm{REG}}$ cells into the liver and its absence causes fulminant liver failure with strongly reduced survival. ${ }^{15}$ Thus, the function of CXCR3 during acute liver injury appears to be very context and model dependent and includes beneficial as well as harmful effects.

Systemic application of the hepatotoxin carbon tetrachloride $\left(\mathrm{CCl}_{4}\right)$ mimics toxic liver injury with acute hepatocellular necrosis, which is accompanied by a massive infiltration of immune cells. ${ }^{16}$ We could recently show that chemokines, including the CXCR3 ligand CXCL9, are strongly elevated within the liver after a single injection of $\mathrm{CCl}_{4}{ }^{17}$ However, the impact of different chemokine receptors present on T, NK and NKT cells in the disease process has not yet been systematically analyzed in this model. Nevertheless, this seems of great clinical importance as NK and NKT cells have been reproducibly shown to be functionally relevant in acute liver disease models. ${ }^{18-20}$ Therefore, we here analyze the specific role of CXCR3 and its impact on NK and NKT cell recruitment during the early phases of toxic liver damage.

\section{MATERIALS AND METHODS Animals}

$\mathrm{CXCR}^{-1-}$ mice were established as described before and were kept on the C57BL/6 background. ${ }^{21}$ Wild-type, CCR $1^{-1-}$ and $\mathrm{CCR}^{-1-}$ mice on the C57BL/6 background were purchased from Jackson Laboratories (Maine, USA) and bred at the central animal facilities of Aachen University Hospital. In all, 6- to 8-week-old chemokine receptor knockout mice and wild-type animals were challenged with a single dose of $\mathrm{CCl}_{4}(0.6 \mathrm{mg} / \mathrm{kg}$ body weight $)$ intraperitoneally (i.p.) to induce acute toxic liver injury. Animals were killed 24 and $72 \mathrm{~h}$ after the $\mathrm{CCl}_{4}$ injection. For histological assessment of liver injury, paraffin-embedded liver sections were stained with $\mathrm{H} \& \mathrm{E}$ and serum transaminases (AST/ALT) were measured as described. ${ }^{17}$

All animals were housed under specific pathogen-free conditions and the experiments were carried out after approval by the animal welfare board at the Bezirksregierung Cologne, Germany.

\section{FACS Analysis of Intrahepatic Immune Cell Subsets}

Explanted livers were perfused with PBS, incubated in collagenase (Roche, Mannheim, Germany) at $37^{\circ} \mathrm{C}$ for $45 \mathrm{~min}$ and then homogenized. After density gradient centrifugation with Lymphocyte Mammal (Cedarlane, Canada), the middle layer, comprising the intrahepatic mononuclear cells, was transferred into a Falcon tube and washed twice with Hank's balanced salt solution (BSS; PAA Laboratories, Austria). Anti-CD45-APC-C7, anti-NK1.1-PE and anti-CD3-APC antibodies (all from BD Biosciences) were diluted 1:200 in blocking buffer and transferred to the isolated cells. The suspension was incubated for $20 \mathrm{~min}$ at $4{ }^{\circ} \mathrm{C}$ in the dark. The cells were washed with Hank's BSS, fixed in 2\% formalin and than analyzed by flow cytometry (FACS Canto II, BD Biosciences). Data were analyzed using the FlowJo program (http://www.flowjo.com).

\section{Gene and Protein Expression Analysis}

Total RNA was isolated from livers of mice and reversely transcribed using Super-Script (Invitrogen, Germany). Quantitative RT-PCR was carried out for IL1- $\beta$, TNF- $\alpha$ and Perforin (PRF1) by assays on demand from http://www. appliedbiosystems.com. IFN- $\gamma$ was quantified by ELISA (RD Systems, Germany) from total liver protein isolates.

\section{TUNEL Assay}

Liver cryosections were fixed in $4 \%$ paraformaldehyde for $20 \mathrm{~min}$ at room temperature (RT). After $30 \mathrm{~min}$ of washing in PBS, the sections were treated with $3 \% \mathrm{H}_{2} \mathrm{O}_{2}$ in methanol for $10 \mathrm{~min}$ at RT and with $0.1 \%$ Triton in $0.1 \%$ sodium citrate for $2 \mathrm{~min}$ at $4{ }^{\circ} \mathrm{C}$. The sections were incubated for $1 \mathrm{~h}$ in TUNELMix (Roche) and photomicrographs were taken with a fluorescence microscope (Zeiss, Germany). ImageJ (http:// rsbweb.nih.gov/ij) was used to count the TUNEL-positive cells per view field.

\section{NK Cell Depletion}

Wild-type mice were injected with $50 \mu \mathrm{l}$ rabbit anti-mouse ASIALO GM1 antibody (Cedarlane) i.p. $1 \mathrm{~h}$ before $\mathrm{CCl}_{4}$ administration. Animals were killed 24 and $72 \mathrm{~h}$ after the $\mathrm{CCl}_{4}$ injection and the livers were analyzed by FACS as described above. For determination of the degree of liver

Figure 1 (a) Serum levels of alanine aminotransferase (ALT) in C57BL/6 wild-type mice (WT; white bars) and CXCR3 ${ }^{-1-}$ mice (black bars) at baseline as well as 24 and $72 \mathrm{~h}$ after injury ( $n=10-12 /$ group). $\mathrm{CXCR3}^{-1-}$ animals have significantly higher ALT values compared with wild-type mice $24 \mathrm{~h}$ after $\mathrm{CCl}_{4}$ injection. Results are shown as mean values \pm s.e.m. $\left({ }^{*} P<0.05\right)$. Please note the logarithmic scale. (b) Representative liver histology (H\&E staining; magnification $\times 200$ ) of C57BL/6 wild-type mice (left) and CXCR3 ${ }^{-1-}$ mice (right) at baseline as well as 24 and $72 \mathrm{~h}$ after liver injury. We observed no histological differences between wild-type and $\mathrm{CXCR}^{-1-}$ mice at baseline. However, $\mathrm{CXCR} 3^{-1-}$ mice show enlarged areas of liver damage (marked with black lines) $24 \mathrm{~h}$ after $\mathrm{CCl}_{4}$-induced liver injury compared with corresponding wild-type mice. These enlarged areas of injury persist in $\mathrm{CXCR}^{-1-}$ mice $72 \mathrm{~h}$ after $\mathrm{CCl}_{4}$ administration, while wild-type mice have completely recovered at this time. 

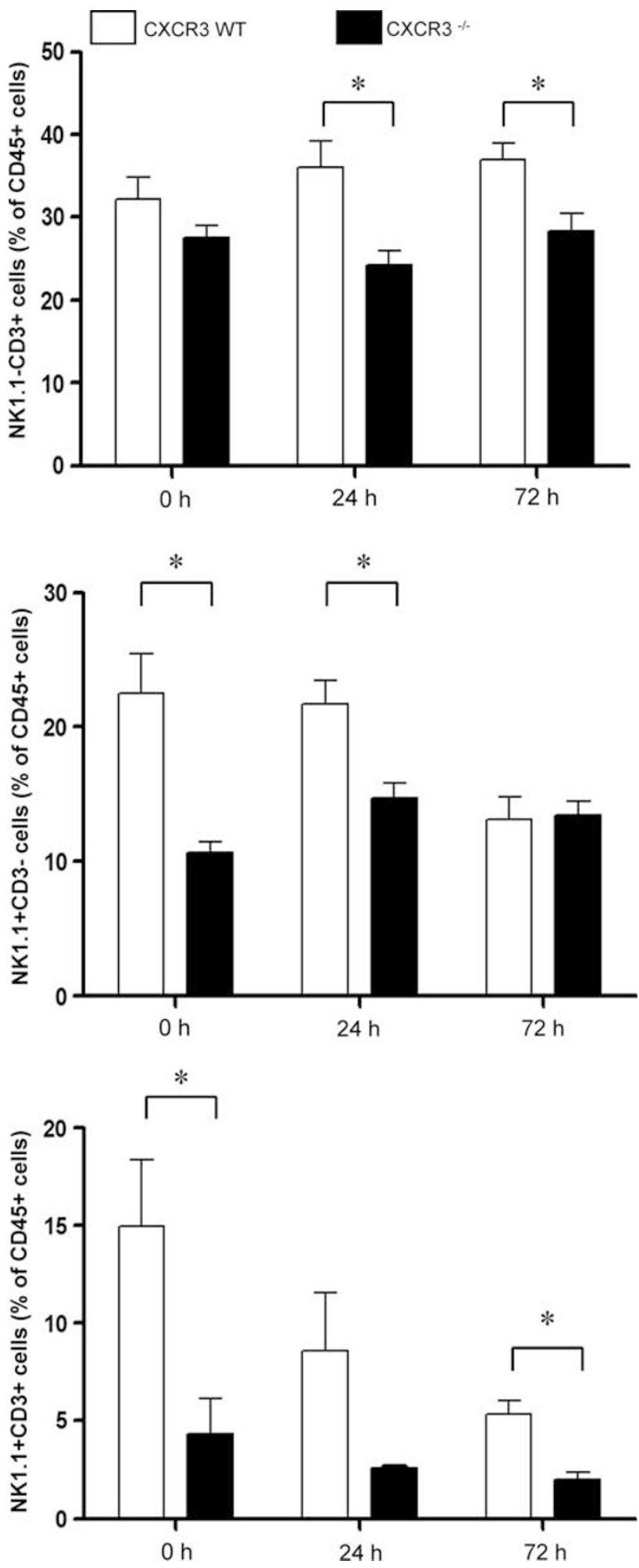

Figure 2 FACS analysis of infiltrating immune cells within the livers of C57BL/ 6 wild-type mice (white bars) and $\mathrm{CXCR}^{-/-}$mice (black bars) with and without administration of $\mathrm{CCl}_{4}(n=10-12 /$ group). Results are shown as mean values \pm s.e.m. $\left({ }^{*} P<0.05\right)$. The FACS data for T cells $(N K 1.1-C D 3+)$, NK cells $(\mathrm{NK} 1.1+\mathrm{CD} 3-)$ and NKT cells $(\mathrm{NK} 1.1+\mathrm{CD} 3+)$ are depicted in the panels. injury, serum transaminases were measured and H\&E staining was performed on paraffin-embedded liver sections. ${ }^{17}$

\section{Histological Quantification of Neutrophils and Macrophages}

In all, $5 \mu \mathrm{m}$ thick cryosections from the livers of treated animals were fixed in $4 \%$ paraformaldehyde for $20 \mathrm{~min}$ at RT. After washing, the slides were incubated overnight at $4{ }^{\circ} \mathrm{C}$ with anti-Ly6G antibody (Invitrogen). The slides were mounted with DAPI $45 \mathrm{~min}$ after incubation with Alexa Flour 488 conjugated secondary antibody (Invitrogen) at RT. Photomicrographs were taken with a fluorescence microscope (Zeiss) and Ly6G + cells (green) per view field were counted using ImageJ.

The numbers of macrophages were assessed at baseline and different time points after injury by staining of paraffinembedded mouse section with a rat anti-mouse F4/80 antibody (Clone BM8; Dianova, Germany). Positive cells were assessed semiquantitatively by two independent investigators.

\section{Naphtol AS-D Chloroacetate Esterase Stain}

In all, $5 \mu \mathrm{m}$ paraffin-embedded liver sections were stained for chloroacetate esterase using the Naphtol AS-D chloroacetate esterase kit (Sigma, Germany) to further assess the infiltration of neutrophils when no liver cryosections were available. The photomicrographs were taken by light microscopy and neutrophils were counted per view field.

\section{High Mobility Group 1 Protein Western Blot}

Snap-frozen liver samples were incubated in $1 \mathrm{ml}$ RIPA buffer (20 mM Tris- $\mathrm{HCl}, 150 \mathrm{mM} \mathrm{NaCl}, 2 \%$ Nonident P40, $0.1 \%$ SDS, $0.5 \%$ Na-deoxycholate) with protease inhibitor (Mini complete Protease Inhibitor Cocktail; Roche) for $30 \mathrm{~min}$ on ice for isolation of total liver protein. For western blotting, each lane was mounted with $30 \mu \mathrm{g}$ total liver protein. After blotting for $2 \mathrm{~h}$, the Protran nitrocellulose membrane (Whatman Schleicher \& Schuell, Germany) was blocked for $1 \mathrm{~h}$ and incubated overnight at $4{ }^{\circ} \mathrm{C}$ with a polyclonal rabbit anti-mouse HMGB1 antibody (eBiosciences, Germany). After incubation with a goat anti-rabbit-HRP (DAKO, Germany), the chemoluminescence reaction was initialized with ECL (Thermo Scientific, Germany) and visualized with the LAS reader 300 (Fujiflim, Germany). $\beta$-Actin was used as loading control. For quantification of the intrahepatic high mobility group 1 (HMGB1) content, the program Quantity One (Bio-Rad Laboratories, http://www.bio-rad.com) was used.

\section{Statistical Analysis}

All data were analyzed using GraphPad Prism Software (GraphPad Software, La Jolla, CA, USA). The results are presented as mean values \pm standard error of the mean (s.e.m.). Continuous variables were compared with Student's $t$-test. $P$-values $<0.05$ were considered as statistically significant. 

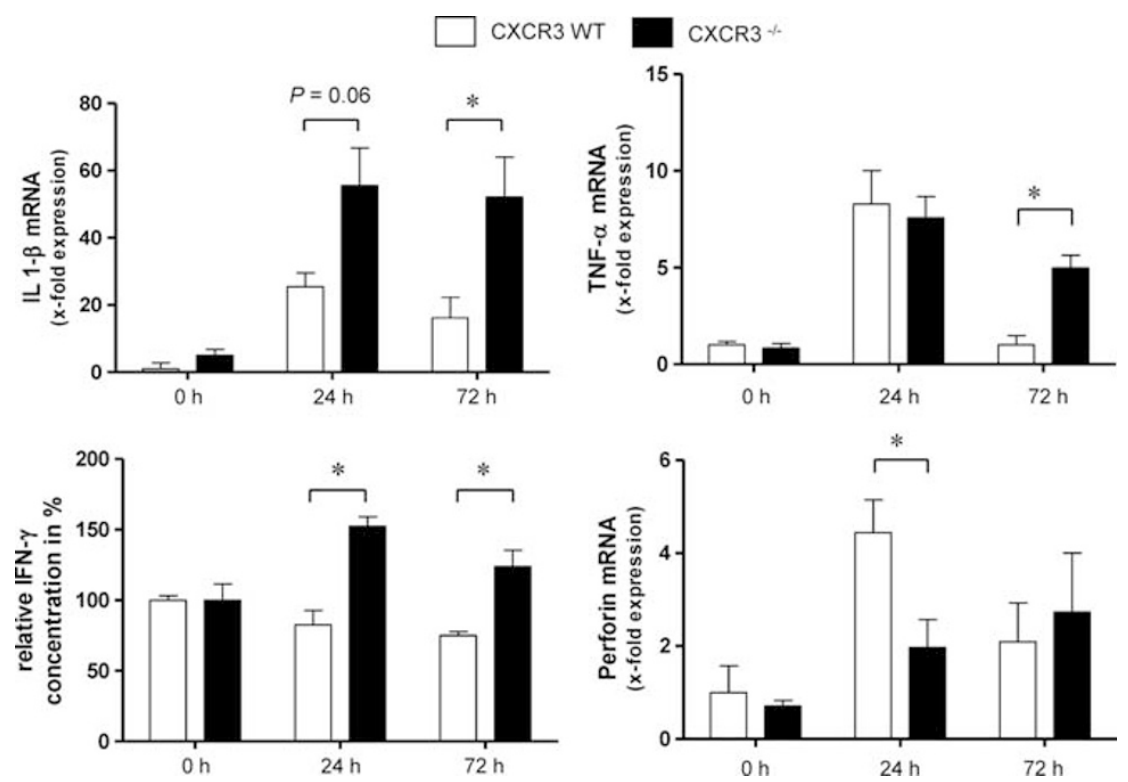

Figure 3 mRNA expression of pivotal proinflammatory genes and the cytotoxic protein perforin in the livers of C57BL/6 wild-type mice (white bars) and $\mathrm{CXCR3}^{-1-}$ mice (black bars, $n=6-8 /$ group). Overall, $\mathrm{CXCR}^{-1-}$ mice display increased mRNA expression of all cytokines but a reduced mRNA level of perforin, which is in line with the reduced infiltration of NK cells. ${ }^{\star} P<0.05$.

\section{RESULTS}

\section{CXCR3 Deficient Mice Display Severe Liver Damage after $\mathrm{CCl}_{4}$ Injection}

We first subjected $\mathrm{CXCR}^{-l-}$ and wild-type mice to acute liver injury by a single injection of $\mathrm{CCl}_{4}$. In comparison with wild-type mice, CXCR3 deficient animals displayed strongly increased liver damage in this experimental model, confirming data of these mice in the ConA model of liver injury. ${ }^{15}$ Augmented injury was reflected by significantly increased ALT (Figure 1a) and AST (data not shown) levels $24 \mathrm{~h}$ after $\mathrm{CCl}_{4}$ administration, while there was no obvious difference between the mouse strains after $72 \mathrm{~h}$. Liver histology confirmed the augmented liver injury in CXCR3 ${ }^{-1-}$ mice (Figure 1b). Compared with their wild-type counterparts, CXCR3 ${ }^{-1-}$ mice showed strongly enlarged areas of pericentral hepatocyte damage $24 \mathrm{~h}$ after $\mathrm{CCl}_{4}$ challenge. Furthermore, the CXCR3 deficient animals still displayed necrotic areas during the recovery phase $72 \mathrm{~h}$ after injury, while wild-type mice had visually and biochemically almost completely recovered at this time point.

\section{Genetic Deletion of CXCR3 Results in an Impaired Recruitment of Immune Cells and an Augmented Expression of Proinflammatory Cytokines}

CXCR3 is considered as an important receptor for recruitment of immune cells to sites of tissue damage. Specifically, ligands of CXCR3 are among the main chemoattractants for NK and NKT cells into the liver. ${ }^{11}$ Since these cells appear to have essential roles in the phenotype of liver damage in different experimental injury models, ${ }^{15,20,22}$ we performed in-depth FACS analysis for intrahepatic T cells (NK1.1-,
$\mathrm{CD} 3+)$, NK cells $(\mathrm{NK} 1.1+, \mathrm{CD} 3-)$ and NKT cells $(\mathrm{NK} 1.1+, \mathrm{CD} 3+)$ during the first $72 \mathrm{~h}$ after $\mathrm{CCl}_{4}$ administration. As depicted in Figure 2, untreated $\mathrm{CXCR}^{-1-}$ animals showed no difference in the frequency of $\mathrm{T}$ cells compared with wild-type mice, while 24 and $72 \mathrm{~h}$ after $\mathrm{CCl}_{4}$ treatment $\mathrm{CXCR}^{-1-}$ mice had fewer $\mathrm{CD} 3+\mathrm{T}$ cells $(P<0.05)$. In contrast to $\mathrm{T}$ cells, untreated $\mathrm{CXCR}^{-1-}$ mice had already significantly lower numbers of NK cells than wild-type mice at baseline $(P<0.05)$, a difference which remained stable $24 \mathrm{~h}$ after induction of liver injury. A strong difference between $\mathrm{CXCR} 3^{-1-}$ mice and wild-type mice was further observed for NKT $(\mathrm{CD} 3+\mathrm{NK} 1.1+)$ cells. CXCR3 deficient mice already display a reduced intrahepatic number of NKT cells before liver injury, a finding which is consistent with earlier findings of the same mice ${ }^{6}$ and the important role of the CXCR3 ligand CXCL9 for NKT cell trafficking to the liver. ${ }^{11}$ Notably, the strongly reduced number of NKT cells in $\mathrm{CXCR}^{-1-}$ mice remained stable throughout the whole observation period after induction of toxic liver injury and its recovery phase.

The observed differences in immune cell recruitment were paralleled by strong alterations in the mRNA expression of pivotal proinflammatory cytokines in $\mathrm{CXCR}^{-1-}$ mice. Specifically, the mRNA levels of IL1- $\beta$ and TNF- $\alpha$ as well as IFN- $\gamma$ protein levels were increased in the $\mathrm{CXCR}^{-1-}$ mice compared with wild-type mice (Figure 3). Conversely, the mRNA expression of perforin, a mediator of NK cell cytotoxicity, ${ }^{23}$ was reduced in CXCR3 ${ }^{-1-}$ mice compared with their wild-type littermates (Figure 3). This finding further suggested a functional role of NK cells in the phenotype observed in our model. 


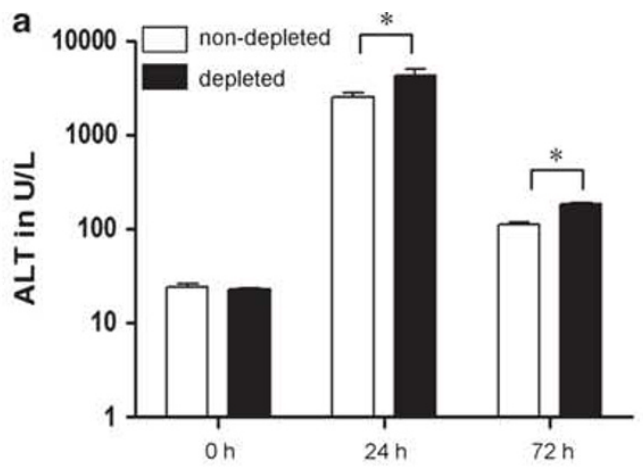

b
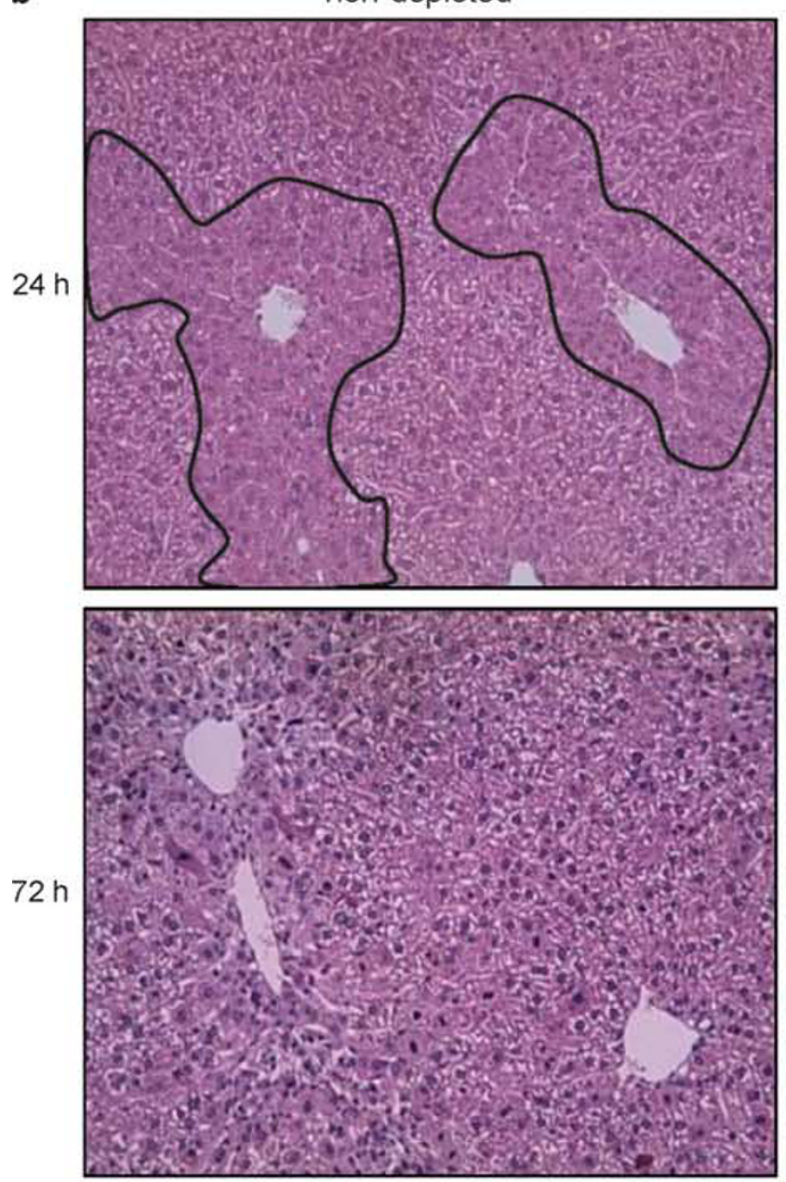

depleted
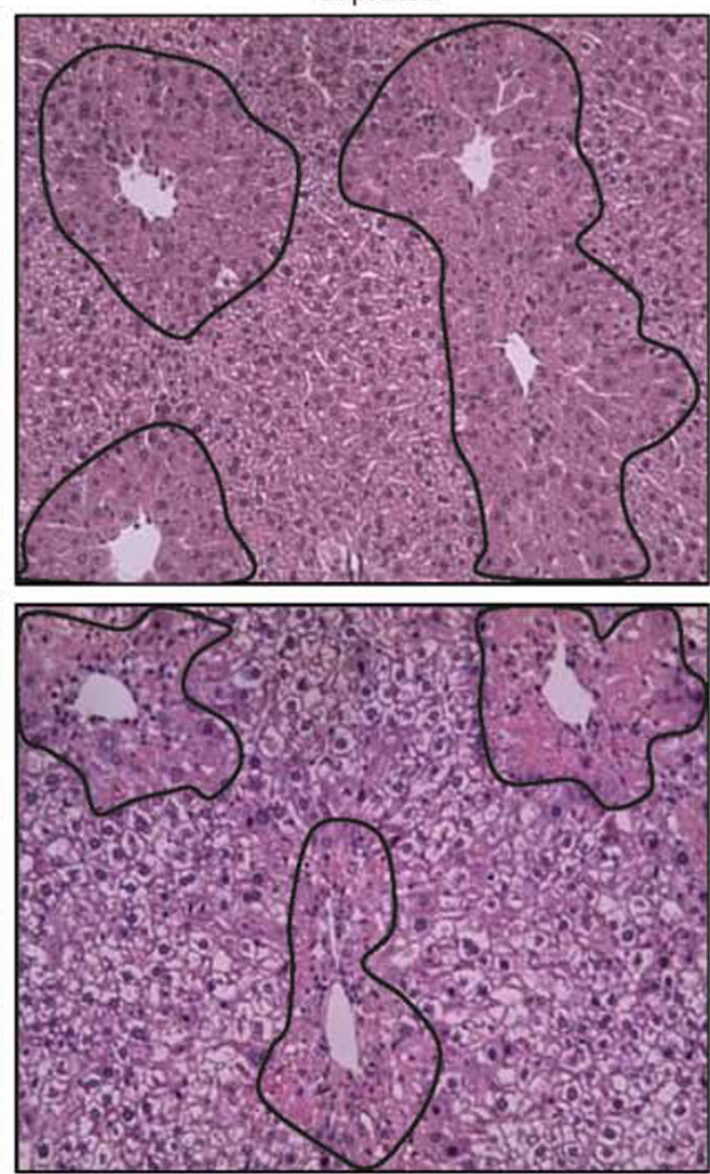

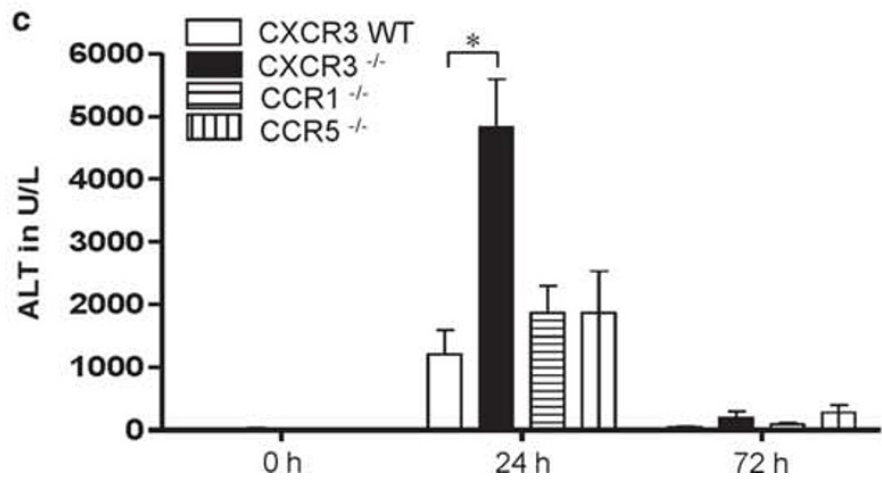

Figure 4 For caption see page 730. 


\section{Depletion of NK and NKT Cells Aggravates $\mathrm{CCl}_{4}$-Induced Liver Damage}

In the light of these data, we next tested whether depletion of NK cells could aggravate liver injury after $\mathrm{CCl}_{4}$ administration. Complete depletion of NK cells and partial depletion of NKT cells were achieved by administration of a single dose of the anti-ASIALO GM1 antibody (Supplementary Figure 1) before challenging the mice with $\mathrm{CCl}_{4}$.

Depletion of these immune cells indeed led to an augmented injury response compared with mice injected with a control antibody. Increased liver damage of mice injected with the anti-ASIALO GM1 antibody compared with nondepleted mice was again reflected by increased serum ALT values 24 and $72 \mathrm{~h}$ after $\mathrm{CCl}_{4}$ administration, respectively $(P<0.05$, Figure $4 a)$. Increased serum transaminases resulted from enlarged areas of hepatic necrosis in depleted mice compared with non-depleted control mice (Figure 4b).

\section{CCR1 and CCR5 Deficient Mice Display no Differences in Liver Injury and Recruitment of NK Cells Compared with Wild-Type Mice in the $\mathrm{CCl}_{\mathbf{4}}$ Model}

As the chemokine receptors CCR1 and CCR5 are also present on NK cells, ${ }^{24}$ we next assessed the specificity of our findings for the chemokine receptor CXCR3. As shown in Figure 4c, mice deficient in CCR1 and CCR5 displayed no significant differences in serum ALT levels 24 and $72 \mathrm{~h}$ after $\mathrm{CCl}_{4}$ administration compared with wild-type mice. Interestingly, FACS analysis revealed that naive CCR $1^{-1-}$ and CCR $5^{-/-}$ mice also have significantly less intrahepatic NK cells compared with untreated wild-type mice (both $P<0.001$, Supplementary Figure 2). However, in contrast to CXCR3 ${ }^{-1-}$ mice, CCR $1^{-l-}$ and $\mathrm{CCR}^{-1-}$ mice show a strong NK cell recruitment into the liver $24 \mathrm{~h}$ after $\mathrm{CCl}_{4}$ administration. Furthermore, there were no differences in NK frequencies between wild-type, $\mathrm{CCR}^{-1-}$ and $\mathrm{CCR}^{-1-}$ mice $72 \mathrm{~h}$ after the $\mathrm{CCl}_{4}$ injection. Thus, the reduced number of $\mathrm{NK}$ and NKT cells in $\mathrm{CXCR}^{-1-}$ mice and especially the lack of recruitment of these cells into the liver after administration of $\mathrm{CCl}_{4}$ appear to be responsible for the increased liver damage observed in these mice.

\section{$\mathrm{CXCR3}^{-1-}$ Mice Display an Increased Neutrophil Recruitment Via HMGB1}

We next assessed which molecular mediators could be responsible for the increased damage in $\mathrm{CXCR}^{-1-}$ and $\mathrm{NK}$ cell-depleted mice. A candidate molecule is HMGB1 protein that is released from necrotic, but not apoptotic, liver cells. ${ }^{25}$ HMGB1 modulates the recruitment of neutrophils into the liver, ${ }^{26}$ where they mediate acute liver damage under different experimental conditions. ${ }^{20,27,28}$

In accordance with these data, we indeed detected increased levels of HMGB1 (Figure 5a), but fewer apoptotic cells (Figure $5 \mathrm{~b}$ ) in livers of CXCR3 ${ }^{-1-}$ and NK cell-depleted mice compared with their wild-type littermates after the toxic insult with $\mathrm{CCl}_{4}$. In line with the higher concentrations of HMGB1 in $\mathrm{CXCR}^{-1-}$ and depleted mice, these animals indeed had an increased number of intrahepatic Ly6G+ neutrophils as compared with wild-type mice after induction of liver injury (Figure 6a; Supplementary Figure 3). As HMGB1 has also been shown to be involved in macrophage recruitment and activation, ${ }^{29}$ we also assessed the number of macrophages (F4/80-positive cells) in our animal models. However, apart from a slightly more pronounced perivascular staining, there were no major differences in the numbers of macrophages in the $\mathrm{CXCR}^{-1-}$ or the NK celldepleted mice compared with wild-type animals during the course of liver injury (Supplementary Figure 4). Thus, neutrophil infiltration secondary to increased HMGB1 concentrations appears to be the main functional consequence of CXCR3 knockdown or NK cell depletion in our acute toxic liver injury model.

\section{DISCUSSION}

The data presented in this study suggest that the chemokine receptor CXCR3 mediates non-redundant beneficial effects during acute liver injury induced by systemic application of the hepatotoxin $\mathrm{CCl}_{4}$. These results are in line with our earlier findings in chronic liver injury models ${ }^{21}$ and a recent report of increased mortality of CXCR $3^{-1-}$ mice after ConAinduced liver damage. ${ }^{15}$ However, there are also studies of ischemia-reperfusion injury and virus-induced liver injury that suggests harmful effects of the receptor in vivo. ${ }^{13,14}$ Thus, the overall properties of CXCR3 in the liver appear to be strongly dependent on the type of liver injury and the experimental conditions applied in different studies. In the current study, we used a model of acute liver injury that results in hepatocyte necrosis and subsequent infiltration of leukocytes from peripheral blood. ${ }^{16,20}$ Notably, in this model, we have already noted a strong induction of the CXCR3 ligand CXCL9 within the liver, ${ }^{17}$ which was identified as a

Figure 4 (a) Serum levels of alanine aminotransferase (ALT) in NK cell-depleted C57BL/6 wild-type mice (undepleted: white bars; depleted: black bars) at baseline as well as 24 and $72 \mathrm{~h}$ after $\mathrm{CCl}_{4}$ injection. The NK cell-depleted animals have significantly higher transaminases 24 and $72 \mathrm{~h}$ after the $\mathrm{CCl}_{4}$ treatment compared with undepleted animals ( $n=5 /$ group). Results are shown as mean values \pm s.e.m. $\left({ }^{*} P<0.05\right.$ ). Please note the logarithmic scale. (b) Representative liver histopathology (H\&E staining; magnification $\times 200$ ) of NK cell-depleted and undepleted C57BL/6 wild-type mice 24 and $72 \mathrm{~h}$ after liver injury. NK cell-depleted animals display larger areas of liver damaged compared with the undepleted animals at both time points. (c) Transaminases of C57BL/ 6 wild-type, $\mathrm{CXCR}^{-1-}$ mice, $\mathrm{CCR}^{-1-}$ mice and CCR5 ${ }^{-1-}$ mice before as well as 24 and $72 \mathrm{~h}$ after $\mathrm{CCl}{ }_{4} \mathrm{Challenge.} \mathrm{CXCR} 3^{-1-}$ mice have significantly higher transaminases $24 \mathrm{~h}$ after the $\mathrm{CCl}_{4}$ injection compared with wild-type mice, while CCR $1^{-/-}$mice and $\mathrm{CCR} 5^{-/-}$mice show similar transaminases as wild-type mice at all time points. ( $n=6-12 /$ group). Results are shown as mean values \pm s.e.m. ( ${ }^{\star} P<0.05$ ). 
a
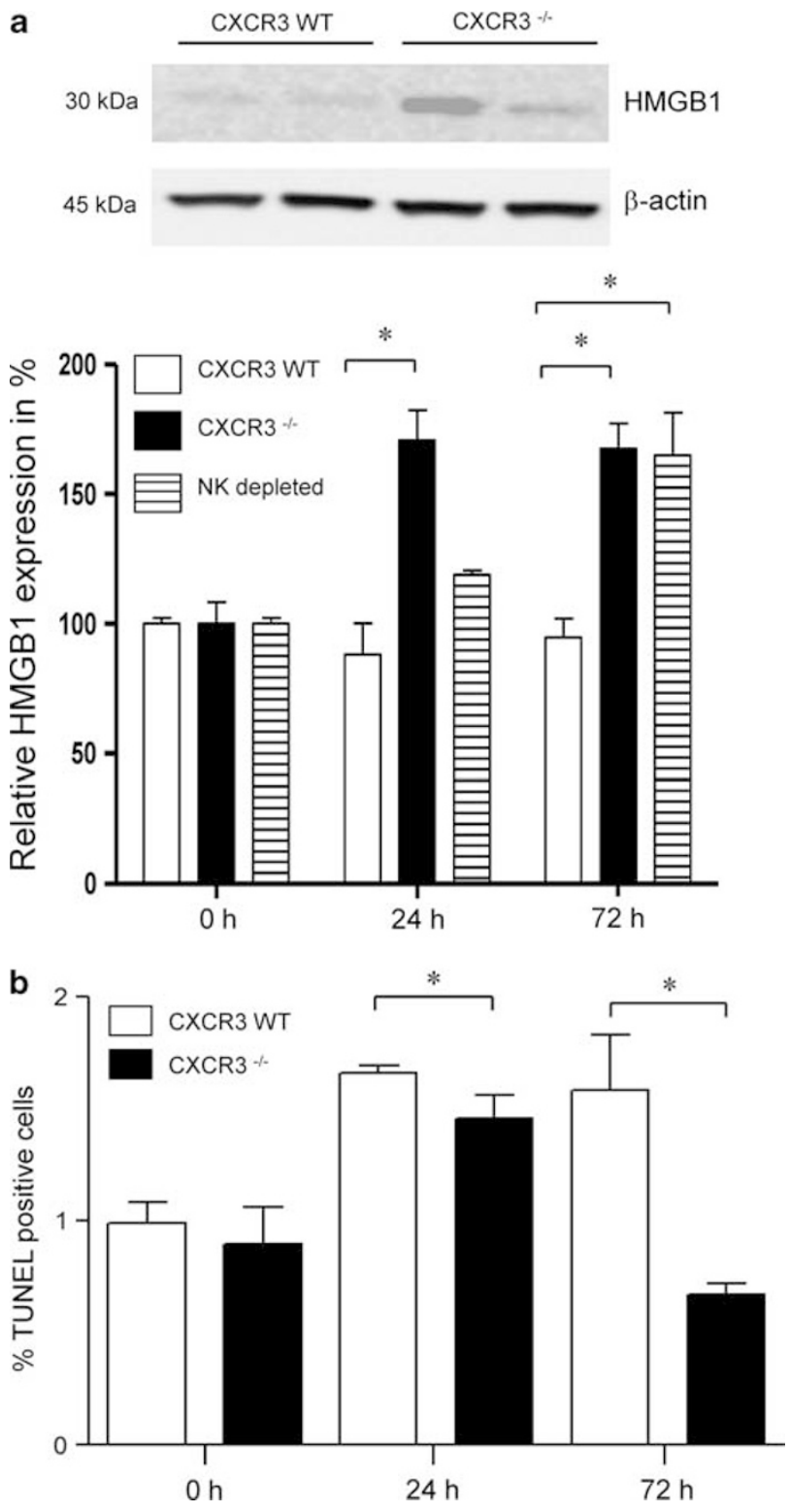

Figure 5 HMGB1 protein expression of liver lysates from wild-type, $\mathrm{CXCR3}^{-1-}$ and NK cell-depleted mice 24 and $72 \mathrm{~h}$ after $\mathrm{CCl}_{4}$ treatment. (a) Representative western blot for HMGB1 expression in wild-type and $\mathrm{CXCR3}^{-1-}$ mice (upper panel) and quantification of intrahepatic HMGB1 protein levels from wild-type mice, $\mathrm{CXCR}^{-1-}$ mice and NK cell-depleted mice after $\mathrm{CCl}_{4}$ treatment ( $n=5 /$ group) as relative expression compared with untreated mice (lower panel). Results are shown as mean values \pm s.e.m. $\left({ }^{*} P<0.05\right)$. (b) Quantification of apoptotic liver cells by TUNEL assay from cryosections of livers of wild-type mice (white bars) and $\mathrm{CXCR3}^{-1-}$ mice (black bars) before and after $\mathrm{CCl}_{4}$ treatment ( $n=5 /$ group). In all, 24 and $72 \mathrm{~h}$ after the $\mathrm{CCl}_{4}$ administration the $\mathrm{CXCR}^{-1-}$ mice have a significant lower number of TUNEL-positive cells despite increased hepatic damage. Results are shown as mean values \pm s.e.m. $\left({ }^{*} P<0.05\right)$. major chemokine for hepatic recruitment of NK and NKT cells ${ }^{11}$ by mediating their transendothelial migration. ${ }^{30}$ As we observed that $\mathrm{CXCR}^{-I-}$ mice were more prone to severe liver damage in the $\mathrm{CCl}_{4}$ model, we thus assessed whether this phenotype is associated with an altered recruitment of $\mathrm{T}$, NK and NKT cells into the liver. Indeed, a dominant immune phenotype of the CXCR $3^{-/-}$mice used in our study was the reduced NK and NKT cell recruitment after the toxic insult (Figure 2), together with a reduced mRNA expression of perforin, a major cytolytic protein of these cells (Figure 3 ). This immune phenotype is consistent with the role of CXCR3 ligands for immune cell recruitment ${ }^{11}$ and earlier findings of hepatic immune cell populations in these mice. ${ }^{6}$ Additionally, CXCR3 has been shown to mediate the infiltration of $\mathrm{T}_{\text {REG }}$ into the liver, ${ }^{12}$ which appears to have a functional role in ConA-induced liver damage. ${ }^{15}$ However, the specific role of $\mathrm{T}_{\mathrm{REG}}$ cells in $\mathrm{CCl}_{4}$-mediated liver injury is not yet decided and in contrast to other immune cell sub-populations these cells represent only a minor fraction of liver leukocytes. We therefore did not further investigate this specific cell type in our model. In contrast, NK and NKT cells are more abundant within the human and murine liver. ${ }^{31,32}$ Notably, genetic depletion of NKT cells has recently been shown to be detrimental in acute $\mathrm{CCl}_{4}$-induced liver injury. ${ }^{20}$ Thus, it appears that the relative lack of NKT cells in the $\mathrm{CXCR}^{-1-}$ mice might functionally be involved in the augmented liver damage compared with wild-type mice.

Another cell population that was strongly reduced in the livers of $\mathrm{CXCR}^{-l-}$ mice are NK cells. These cells are known to be involved in liver injury ${ }^{33}$ where they mediate apoptosis of different liver resident cells. ${ }^{34,35}$ However, their specific role in acute $\mathrm{CCl}_{4}$-mediated liver injury has not yet been elucidated. As NK cells might also be responsible for the increased liver damage in CXCR3 $3^{-1-}$ mice, we depleted these cells with an anti-mouse ASIALO GM1 antibody in wild-type mice before liver injury. Indeed, depleted mice displayed increased liver damage as assessed by histology and serum transaminases (Figure 4). NK cells do not only express CXCR3, but also the chemokine receptors CCR1 and CCR5. ${ }^{24,36}$ Thus, we also challenged mice with a genetic deletion of these chemokine receptors with $\mathrm{CCl}_{4}$. In contrast to the $\mathrm{CXCR}^{-/-}$mice, these mouse strains did not display increased liver damage (Figure 4c) and were able to recruit NK cells into the liver (Supplementary Figure 2). Thus, we hypothesize that either a lack of NK cells or a relative inability to recruit these cells to the site of damage is functionally detrimental in the intraperitoneal $\mathrm{CCl}_{4}$ model of liver injury.

Figure 6 (a) Quantification of Ly6G + cells (neutrophils) on cryosections of the livers from $\mathrm{CCl}_{4}$-treated wild-type and CXCR3 $3^{-1-}$ mice ( $n=6-8 / \mathrm{group}$ ). $\mathrm{CXCR3}^{-1-}$ mice have a significantly higher intrahepatic neutrophil count compared with wild types 24 and $72 \mathrm{~h}$ after liver damage. Results are shown as mean values \pm s.e.m. $\left({ }^{*} P<0.05 ;{ }^{* * *} P<0.001\right)$. (b) Representative immunofluorescence Ly6G staining (magnification $\left.\times 200\right)$ on cryosections of the livers from $\mathrm{CCl}_{4}$-treated wild-type and $\mathrm{CXCR}^{-1-}$ mice. 


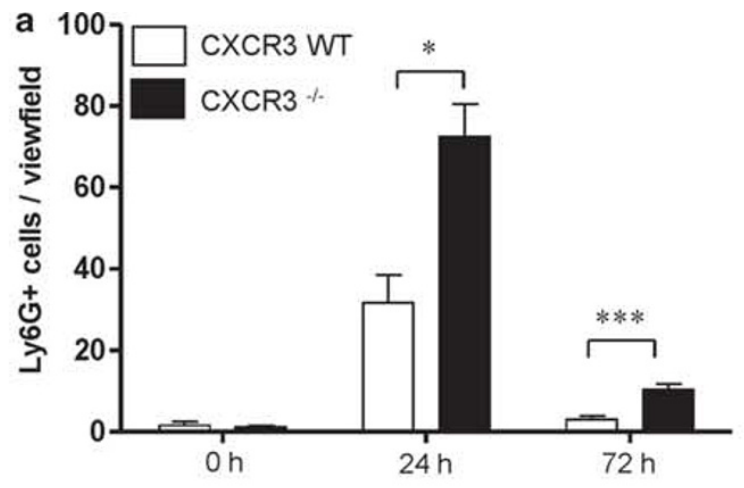

b
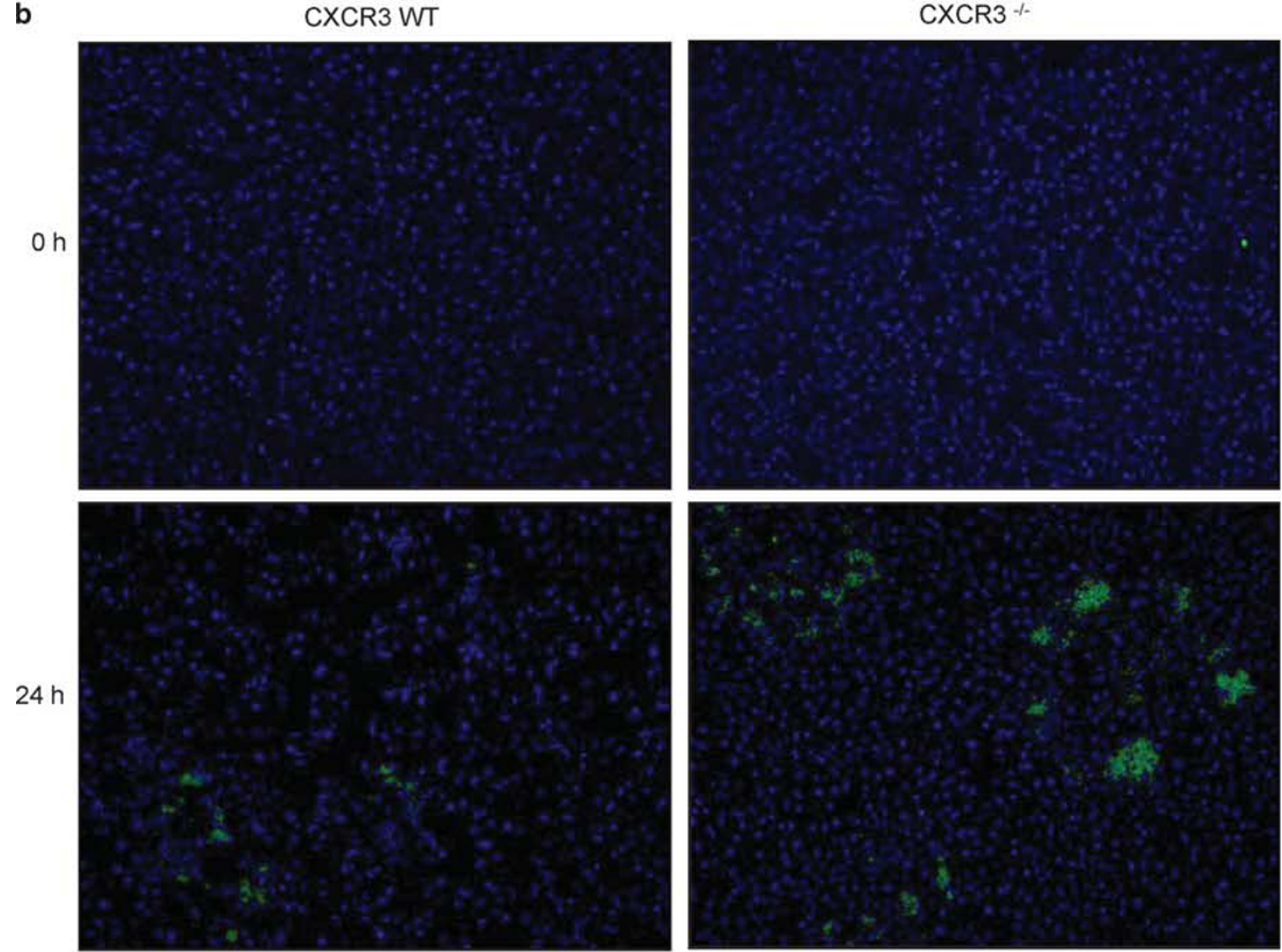

$72 \mathrm{~h}$
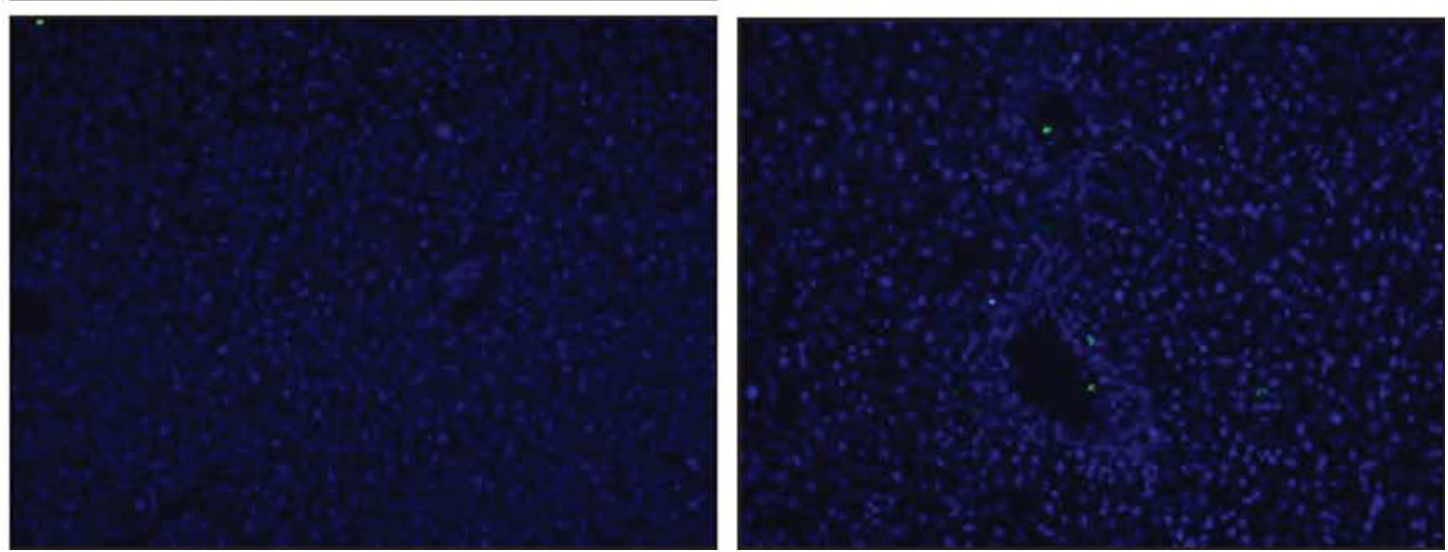

Figure 6 For caption see page 731. 
As NK cells have been associated with apoptotic cell injury, ${ }^{34}$ a TUNEL assay was performed on cryosections of the livers from $\mathrm{CXCR} 3^{-l-}$ and wild-type mice. As depicted in Figure $5 \mathrm{a}, \mathrm{CXCR}^{-1-}$ mice indeed had fewer apoptotic liver cells 24 and $72 \mathrm{~h}$ after $\mathrm{CCl}_{4}$ administration compared with wild-type mice. As $\mathrm{CXCR}^{-1-}$ mice had augmented liver injury, we speculated that most damage might be due to necrotic hepatocyte damage. This hypothesis was indeed supported by higher levels of HMGB1 in the $\mathrm{CXCR}^{-1-}$ mice and the NK cell-depleted mice (Figure 5a). HMGB1 is released from necrotic hepatocytes, ${ }^{37}$ induces the production of proinflammatory cytokines, ${ }^{26}$ leads to recruitment of neutrophils ${ }^{38}$ and is an activator of macrophages. ${ }^{29}$ In line with these data, we observed strongly increased numbers of neutrophils in areas of hepatic necrosis in the $\mathrm{CXCR}^{-1-}$ mice and the animals depleted for NK cells (Figure 6; Supplementary Figure 3). Interestingly, this increased recruitment appears to be specific for neutrophils as the number of F4/80positive macrophages was not different between the mouse strains (Supplementary Figure 4). The pivotal role of neutrophils in liver injury has been shown before in ischemiareperfusion injury, ${ }^{27}$ alcoholic hepatitis ${ }^{39}$ or acetaminophen toxicity. ${ }^{28}$ In the latter model, increased liver damage was also correlated with HMGB1 concentrations and a subsequent higher hepatic infiltration of neutrophils, while neutralization of HMGB1 reduced the recruitment of neutrophils. ${ }^{37}$ This interaction between HMGB1 and neutrophil infiltration does not seem to be specific for the liver as similar results were obtained in a model of acute lung injury. ${ }^{40}$ We acknowledge that our study does only provide functional data in a murine model of acute liver injury and we do not present data on the role of CXCR3 or its ligands in samples from patients with acute liver injury. However, recent data show that CXCR3 is indeed strongly expressed in lymphocytes of subjects with acute liver injury. ${ }^{41}$ Thus, our data might also be of relevance for human acute liver diseases.

In summary, we here present evidence that the chemokine receptor CXCR3 critically regulates the migration of NK and NKT cells during $\mathrm{CCl}_{4}$-induced acute liver injury. Reduced numbers of these cells were associated with reduced hepatocyte apoptosis, but a release of HMGB1 from the necrotic liver cells. HMGB1 in turn recruited neutrophils to the injured liver, thereby mediating augmented damage in this model. Although the relevance of our data for human acute liver injury needs to be proven, these results should be considered when planning human trials with CXCR3 antagonists ${ }^{42}$ which might have hepatotoxic side effects due to the mechanisms described in the current study.

Supplementary Information accompanies the paper on the Laboratory Investigation website (http://www.laboratoryinvestigation.org)

\section{ACKNOWLEDGEMENT}

The study was supported from the Deutsche Forschungsgemeinschaft (SFBTRR57 P08, WA 2557/2-1) and the Else Kröner-Fresenius Stiftung to HEW.

\section{DISCLOSURE/CONFLICT OF INTEREST}

The authors declare no conflict of interest.

1. Lee WM. Etiologies of acute liver failure. Semin Liver Dis 2008;28: $142-152$.

2. Wu Z, Han M, Chen T, et al. Acute liver failure: mechanisms of immunemediated liver injury. Liver Int 2010;30:782-794.

3. Sheth K, Bankey P. The liver as an immune organ. Curr Opin Crit Care 2001;7:99-104

4. Bendelac A, Savage PB, Teyton L. The biology of NKT cells. Annu Rev Immunol 2007;25:297-336.

5. Rossi D, Zlotnik A. The biology of chemokines and their receptors. Annu Rev Immunol 2000;18:217-242.

6. Jiang D, Liang J, Hodge J, et al. Regulation of pulmonary fibrosis by chemokine receptor CXCR3. J Clin Invest 2004;114:291-299.

7. Lacotte S, Brun S, Muller S, et al. CXCR3, inflammation, and autoimmune diseases. Ann NY Acad Sci 2009;1173:310-317.

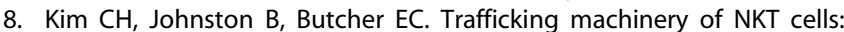
shared and differential chemokine receptor expression among $\mathrm{V}$ alpha 24(+)V beta 11(+) NKT cell subsets with distinct cytokine-producing capacity. Blood 2002;100:11-16.

9. Curbishley SM, Eksteen B, Gladue RP, et al. CXCR 3 activation promotes lymphocyte transendothelial migration across human hepatic endothelium under fluid flow. Am J Pathol 2005;167:887-899.

10. Bonacchi A, Romagnani $P$, Romanelli RG, et al. Signal transduction by the chemokine receptor CXCR3: activation of Ras/ERK, Src, and phosphatidylinositol 3-kinase/Akt controls cell migration and proliferation in human vascular pericytes. J Biol Chem 2001;276:9945-9954.

11. Johnston $B, \operatorname{Kim} \mathrm{CH}$, Soler D, et al. Differential chemokine responses and homing patterns of murine TCR alpha beta NKT cell subsets. J Immunol 2003;171:2960-2969.

12. Santodomingo-Garzon T, Han J, Le T, et al. Natural killer T cells regulate the homing of chemokine CXC receptor 3-positive regulatory $\mathrm{T}$ cells to the liver in mice. Hepatology 2009;49:1267-1276.

13. Ajuebor MN, Jin Y, Gremillion GL, et al. GammadeltaT cells initiate acute inflammation and injury in adenovirus-infected liver via cytokine-chemokine cross talk. J Virol 2008;82:9564-9576.

14. Zhai $Y$, Shen XD, Hancock WW, et al. CXCR3+CD4+ T cells mediate innate immune function in the pathophysiology of liver ischemia/ reperfusion injury. J Immunol 2006;176:6313-6322.

15. Erhardt A, Wegscheid C, Claass B, et al. CXCR3 deficiency exacerbates liver disease and abrogates tolerance in a mouse model of immunemediated hepatitis. J Immunol 2011;186:5284-5293.

16. Kovalovich K, DeAngelis RA, Li W, et al. Increased toxin-induced liver injury and fibrosis in interleukin-6-deficient mice. Hepatology 2000;31:149-159.

17. Berres ML, Trautwein C, Zaldivar MM, et al. The chemokine scavenging receptor D6 limits acute toxic liver injury in vivo. Biol Chem 2009;390:1039-1045.

18. Zou Y, Chen T, Han M, et al. Increased killing of liver NK cells by Fas/Fas ligand and NKG2D/NKG2D ligand contributes to hepatocyte necrosis in virus-induced liver failure. J Immunol 2010;184: $466-475$.

19. Hou X, Zhou R, Wei $H$, et al. NKG2D-retinoic acid early inducible-1 recognition between natural killer cells and Kupffer cells in a novel murine natural killer cell-dependent fulminant hepatitis. Hepatology 2009;49:940-949.

20. Mariette L, Annie L, Gwendoline N, et al. Invariant Natural Killer T celldeficient mice display increased $\mathrm{CCl}(4)$-induced hepatitis associated with CXCL1 over-expression and neutrophil infiltration. Eur J Immunol 2011;41:1720-1732.

21. Wasmuth HE, Lammert F, Zaldivar MM, et al. Antifibrotic effects of CXCL9 and its receptor CXCR3 in livers of mice and humans. Gastroenterology 2009;137:309-319, 319.e1-3.

22. Wintermeyer $P$, Cheng $C W$, Gehring $S$, et al. Invariant natural killer $T$ cells suppress the neutrophil inflammatory response in a mouse model of cholestatic liver damage. Gastroenterology 2009;136:1048-1059.

23. Chavez-Galan L, Arenas-Del Angel MC, Zenteno $\mathrm{E}$, et al. Cell death mechanisms induced by cytotoxic lymphocytes. Cell Mol Immunol 2009;6:15-25. 
24. Bernardini G, Sciume G, Bosisio D, et al. CCL3 and CXCL12 regulate trafficking of mouse bone marrow NK cell subsets. Blood 2008; 111:3626-3634.

25. Scaffidi P, Misteli T, Bianchi ME. Release of chromatin protein HMGB1 by necrotic cells triggers inflammation. Nature 2002;418:191-195.

26. Ramaiah SK, Jaeschke H. Role of neutrophils in the pathogenesis of acute inflammatory liver injury. Toxicol Pathol 2007;35:757-766.

27. Jaeschke H, Farhood A, Smith CW. Neutrophils contribute to ischemia/ reperfusion injury in rat liver in vivo. FASEB J 1990;4:3355-3359.

28. Liu ZX, Han D, Gunawan B, et al. Neutrophil depletion protects against murine acetaminophen hepatotoxicity. Hepatology 2006;43:1220-1230.

29. Dragomir AC, Laskin JD, Laskin DL. Macrophage activation by factors released from acetaminophen-injured hepatocytes: potential role of HMGB1. Toxicol Appl Pharmacol 2011;253:170-177.

30. Schrage A, Wechsung K, Neumann K, et al. Enhanced $\mathrm{T}$ cel transmigration across the murine liver sinusoidal endothelium is mediated by transcytosis and surface presentation of chemokines. Hepatology 2008;48:1262-1272.

31. Knolle PA, Gerken G. Local control of the immune response in the liver. Immunol Rev 2000;174:21-34.

32. Matsuda JL, Naidenko OV, Gapin L, et al. Tracking the response of natural killer T cells to a glycolipid antigen using CD1d tetramers. J Exp Med 2000;192:741-754.

33. Beraza N, Malato $Y$, Sander LE, et al. Hepatocyte-specific NEMO deletion promotes NK/NKT cell- and TRAIL-dependent liver damage. J Exp Med 2009;206:1727-1737.
34. Ochi $\mathrm{M}$, Ohdan $\mathrm{H}$, Mitsuta $\mathrm{H}$, et al. Liver NK cells expressing TRAIL are toxic against self hepatocytes in mice. Hepatology 2004;39: 1321-1331.

35. Melhem A, Muhanna N, Bishara A, et al. Anti-fibrotic activity of NK cells in experimental liver injury through killing of activated HSC. J Hepatol 2006;45:60-71.

36. Khan IA, Thomas SY, Moretto MM, et al. CCR5 is essential for NK cell trafficking and host survival following Toxoplasma gondii infection. PLoS Pathog 2006;2:e49.

37. Antoine DJ, Williams DP, Kipar A, et al. Diet restriction inhibits apoptosis and HMGB1 oxidation and promotes inflammatory cell recruitment during acetaminophen hepatotoxicity. Mol Med 2010;16:479-490.

38. Park JS, Arcaroli J, Yum HK, et al. Activation of gene expression in human neutrophils by high mobility group box 1 protein. Am J Physiol Cell Physiol 2003;284:C870-C879.

39. Bautista AP. Neutrophilic infiltration in alcoholic hepatitis. Alcohol 2002;27:17-21.

40. Kim JY, Park JS, Strassheim D, et al. HMGB1 contributes to the development of acute lung injury after hemorrhage. Am J Physiol Lung Cell Mol Physiol 2005;288:L958-L965.

41. TrehanPati N, Sukriti S, Geffers R, et al. Gene expression profiles of $T$ cells from hepatitis $E$ virus infected patients in acute and resolving phase. J Clin Immunol 2011;31:498-508.

42. Crosignani S, Missotten M, Cleva C, et al. Discovery of a novel series of CXCR3 antagonists. Bioorg Med Chem Lett 2010;20:3614-3617. 